\title{
Economia Solidária e Reabilitação Vocacional no Campo da Drogadição: possibilidades e limites das práticas atuais'
}

Cooperative Work and Vocational Rehabilitation in the Drug Addiction Treatment: opportunities and limitations of current practices

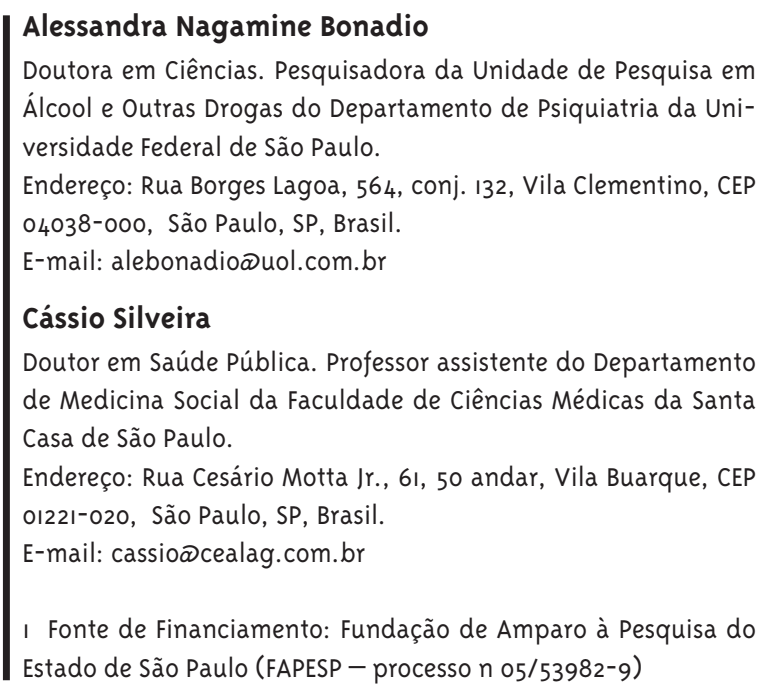

\section{Cássio Silveira}

Doutor em Saúde Pública. Professor assistente do Departamento de Medicina Social da Faculdade de Ciências Médicas da Santa Casa de São Paulo.

Endereço: Rua Cesário Motta Jr., 6I, 50 andar, Vila Buarque, CEP 01221-020, São Paulo, SP, Brasil.

E-mail: cassioœcealag.com.br

I Fonte de Financiamento: Fundação de Amparo à Pesquisa do Estado de São Paulo (FAPESP - processo n 05/53982-9)

\section{Resumo}

Este artigo visa a contribuir ao debate sobre as potencialidades do trabalho no processo de recuperação de dependentes químicos. Consideramos nesta análise os princípios subjacentes à reabilitação vocacional praticada no contexto internacional, seguido da descrição das diretrizes brasileiras para a inclusão social de dependentes químicos por meio do trabalho. Por fim, procedemos a uma análise comparativa das matrizes conceituais, dos conceitos de saúde subjacentes e do potencial emancipatório em ambas as perspectivas. O material consultado foi levantado por meio de revisão bibliográfica em bases de dados da área da saúde. Já as informações sobre as diretrizes brasileiras foram coletadas nas publicações oficiais disponibilizadas on-line pelo Ministério da Saúde e Ministério do Trabalho e do Emprego do Brasil. A análise do material permitiunos verificar que a reabilitação vocacional praticada em países da América do Norte e da Europa destina-se a usuários de serviços de saúde mental, procedendo à inclusão pelo viés da doença. Enfatiza a recolocação no mercado formal de trabalho, por meio de programas voltados ao treinamento de habilidades para obter e manter um posto de trabalho conquistado. Já as diretrizes do governo brasileiro estão pautadas nos princípios do cooperativismo e da economia solidária. Privilegia o ser humano como sujeito e finalidade maior da atividade econômica, focalizando as potencialidades e recursos do trabalho, em detrimento das limitações impostas pela doença ou pela condição socioeconômica que tenha gerado a situação de exclusão. 
Palavras-chave: Transtornos Relacionados ao Uso de Substâncias; Reabilitação Vocacional; Políticas Públicas de Saúde; Trabalho; Readaptação ao Emprego.

\section{Abstract}

This paper contributes to the debate on the potencial of work in the recovery process of addicts. The vocational rehabilitation principles are analyzed and compared to the Brazilian guidelines for the work rehabilitation of this population. In this analysis we compare the underlying concept of health and the emancipatory potential in both perspectives. The literature on vocational rehabilitation was raised through a literature review on Medline, Pubmed and Lilacs, with the following key words: vocational rehabilitation, substance abuse treatment \& work. The Brazilian Government guidelines were collected in the official publications available online by the Ministry of Health and Ministry of Labour and Employment of Brazil. The analysis of the material pointed out that the vocational rehabilitation practiced in North America and Europe focus on users of mental health services, proceeding to the work inclusion through the bias of the disease. The vocational rehabilitation emphasizes the replacement in the formal labor market, through programs aimed at skills training to get and keep a job. On the other hand, the Brazilian public policies are guided by the principles of cooperative and social economy. It privileges the human ethics in its entirety, as the subject and the purpose of higher economic activity, focusing on the strengths and resources of the work, rather than the limitations imposed by the disease or the socioeconomic status that has generated the situation of exclusion.

Keywords: Substance-Related Disorders; Vocational Rehabilitation; Public Health Policy; Work; Supported Employment. 


\section{Introdução}

Usuários de serviços terapêuticos para álcool e drogas historicamente apresentam altos índices de desemprego e grandes dificuldades para conseguir trabalho, durante ou após o período de tratamento (Magura e col., 2004; Effective Interventions Unit, 2001). Este evento pode ser atribuído a diversos fatores, que envolvem as deficiências na formação técnica ou acadêmica dos usuários, a relutância dos empregadores para contratar pessoas com histórico de dependência química, ou mesmo a falta de estímulo ao trabalho que pode decorrer da aquisição de benefícios sociais vinculados à doença e ao desemprego (Magura e col., 2004).

Em pesquisas de avaliação sobre programas de tratamento para a dependência química, a variável trabalho é vista tanto como um resultado desejado - meta a ser atingida -, quanto como um elemento do tratamento (Platt, 1995), vinculada, portanto, a resultados clínicos positivos e à adesão do paciente ao tratamento (National Institute on Drug Abuse, 1999; Platt, 1995). O trabalho favorece tanto a recuperação inicial, como a manutenção da abstinência e a redução do consumo de substâncias (Becker e col., 2005; Room, 1998). Por sua relevância ao processo de recuperação, o trabalho deve se constituir como foco de interesse e monitoração do terapeuta, tanto quanto o consumo da substância em si ou os problemas psicológicos e físicos associados à dependência (Edwards e col., 1999). Em estudo qualitativo que avaliou as prioridades da vida cotidiana, relatadas por uma amostra de pessoas em recuperação para dependência química, o trabalho emergiu como uma das prioridades mais apontadas, junto com educação, moradia e relacionamento familiar e social (Laudet e White, 2010).

Assim, o trabalho parece agregar um sentido fundamental ao debate sobre a reabilitação de dependentes químicos. Ainda que a sua centralidade esteja em discussão atualmente, parece inquestionável o quanto esta atividade permeia esferas diversas da vida humana, engendrando a vida social e sendo por ela ao mesmo tempo determinada (Ferretti, 1997). É pelo trabalho que o homem se torna um ser social, distinguindo-se de todas as formas não humanas e possibilitando a própria produção e reprodução de sua humanidade (Antunes, 200o). Seja nas atividades remuneradas, seja em outras formas possíveis de sua realização, o trabalho desempenha um papel central na conquista de um sentimento de dignidade social. Favorece a aquisição de um senso de propósito e significado na vida e contribui para aumentar a autoestima e para estruturar o cotidiano, ao promover a participação no contexto social, o contato com os semelhantes e o engajamento em uma atividade produtiva (Becker e col., 2005; Comerford, 1999; Room, 1998).

0 debate sobre as potencialidades relacionadas ao eixo do trabalho no processo de recuperação de dependentes químicos levou-nos a uma reflexão mais ampla sobre a questão da dependência química e suas possíveis estratégias de tratamento e reabilitação dos dependentes. Não pretendemos, aqui, trazer o extenso debate já produzido pelas Ciências Sociais sobre tal conceito. Tomamos tão somente emprestada à noção de trabalho a sua essência como processo primordial de realização da vida, que valoriza a unidade concepção-execução como eixo da realidade de uma atividade essencialmente humana (Braverman, 1981). Assim, chamamos a atenção dos leitores para um aspecto derivado de todo o debate sobre trabalho, que coloca a atividade de trabalho, enquanto ocupação, como caminho possível às estratégias de reabilitação dos dependentes químicos.

Numa incursão pela literatura sobre a temática da recuperação no campo da dependência química, constatou-se a afirmação de que pessoas prejudicadas por alguma condição clínica ou social são capazes de desenvolver atividades de trabalho. Mowbray e colaboradores (1997) são enfáticos ao afirmar que todos os indivíduos, independentemente do grau e tipo de comprometimento que apresentem, são capazes de trabalhar produtivamente em qualquer tipo de ambiente, desde que providos de suporte adequado. Segundo os autores, eventuais fracassos no âmbito do trabalho não decorreriam de uma incapacidade pessoal, mas de uma atividade ou ambiente inapropriados, ou de suportes inadequados, o que implicaria trazer a este debate a reflexão mais ampliada sobre o campo de relações sociais envolvidos nas atividades laborais.

No contexto internacional, os serviços de reabilitação vocacional constituem o suporte dispo- 
nibilizado para auxiliar pessoas em tratamento, por diversas condições crônicas, a buscar e manter uma atividade ocupacional. Com este argumento, a chamada reabilitação vocacional é frequentemente vista como um dos meios mais efetivos de se promover o retorno do dependente químico ao mundo do trabalho (Deren e Randell, 1990) e, por consequência, à sociedade. Ao conseguir obter e manter um trabalho, o dependente não apenas estabelece um meio legal de fonte de renda, mas tem melhorada sua autoestima, contribuindo muitas vezes para a redução do consumo de drogas ilícitas e para o afastamento de atividades criminais (Center for Substance Abuse Treatment, 200o).

Consideramos a importância de manter o termo reabilitação vocacional, amplamente utilizado na literatura internacional, referindo-se tão somente aos contextos dos países desenvolvidos, dentro dos quais essas práticas se originaram. Cabe também ressaltar que as concepções e práticas relacionadas à reabilitação vocacional emergem no contexto internacional orientado, política e socialmente, pelo Estado de bem-estar social, comumente denominado welfare state, o que acarreta diferenças significativas em relação às práticas desenvolvidas no contexto brasileiro. Ao considerar as diversas características históricas, políticas e sociais existentes nos países em desenvolvimento, como é o caso do Brasil, afirmamos que as práticas voltadas à reabilitação para o trabalho filiam-se a concepções distintas. No contexto brasileiro, inexistem serviços estruturados para a finalidade de auxiliar o dependente químico no retorno ao trabalho. As iniciativas enfatizadas pelo governo brasileiro pautam-se nos princípios do cooperativismo como forma de promover a inclusão social desta população pelo trabalho (Brasil, 2006a).

Diante deste contexto, o presente artigo visa propor uma discussão sobre a possibilidade de favorecer a recuperação de dependentes químicos pela atividade laboral. Para tanto, foram pesquisados os princípios subjacentes ao processo de reabilitação vocacional praticado no contexto internacional, seguido da descrição das diretrizes brasileiras para a inclusão social de dependentes químicos pelo trabalho. Por fim, procedemos a uma análise comparativa do material bibliográfico e documental levantados, observando as práticas decorrentes de cada matriz conceitual, os conceitos de saúde subjacentes e o potencial emancipatório a elas vinculado.

O processo criativo de nosso estudo exigiu, num primeiro momento, uma incursão na literatura sobre reabilitação vocacional, tarefa realizada mediante uma revisão das principais bases de dados da área da saúde (Medline, Pubmed e Lilacs), efetivada pela escolha dos seguintes descritores na língua inglesa: vocational rehabilitation, substance abuse treatment \& work. Como critério para a seleção do material encontrado, priorizamos a inclusão de artigos com temas conceituais que aprofundassem o debate sobre os princípios e a prática da reabilitação vocacional. De maneira complementar, foram levantadas as informações sobre as diretrizes brasileiras, coletadas nas publicações oficiais, disponibilizadas on-line pelo Ministério da Saúde e Ministério do Trabalho e do Emprego. Dentre as publicações revisadas, foram consultados: relatórios de conferências nacionais sobre saúde mental, portarias oficiais, manuais de programas sociais e de projetos terapêuticos propostos pela política nacional para o tratamento e reabilitação de dependentes químicos, publicados nas três últimas décadas, até o ano de 2010.

\section{Reabilitação Vocacional: uma prática corrente em âmbito internacional}

De maneira genérica, a reabilitação vocacional é descrita como um processo destinado a auxiliar pessoas com comprometimentos de diversas naturezas e graus a retomar e manter uma atividade produtiva, segundo suas possibilidades (Mowbray e col., 1997). No contexto internacional, esse processo é encaminhado por serviços especificamente voltados a essa finalidade, os chamados serviços de reabilitação vocacional, que podem ou não integrar os programas de tratamento, sendo, neste caso, disponibilizados nos próprios settings terapêuticos (Center for Substance Abuse Treatment, 200o).

A população-alvo dos serviços vocacionais inclui os dependentes químicos, mas não se restringe a eles. Portadores de transtornos mentais crônicos também integram frequentemente o quadro de usuários desses serviços (Mowbray e col., 1997). 
O foco desses programas é a empregabilidade e a recolocação no mercado de trabalho. Por isso, os conceitos centrais que embasam as práticas realizadas, assim como as estratégias e os instrumentos utilizados no processo, relacionam-se à empregabilidade, à recolocabilidade, à prontidão para o trabalho e às barreiras ao trabalho, conforme descrito a seguir (Karuntzos, 2002):

1) Empregabilidade: atributo relacionado à capacidade da pessoa de funcionar adequadamente em uma situação particular de trabalho ou numa ocupação específica;

2) Recolocabilidade: probabilidade de uma pessoa conseguir trabalho em uma ocupação específica, considerando-se suas habilidades pessoais;

3) Postura de prontidão ao trabalho: conceito relacionado aos atributos pessoais (motivação, autoestima, desejo e habilidades gerais para obter e manter um emprego) e aos fatores ambientais (preferências do empregador, condições gerais do mercado de trabalho);

4) Barreiras ao trabalho: obstáculos que dificultam a retomada de uma atividade produtiva, podendo ser internos ou externos à pessoa. No âmbito das barreiras internas, figuram: a) aquelas que resultam diretamente da doença (sintomatologia, como ansiedade, fobia social, dificuldades de concentração e memória, entre outras); b) aquelas que são consequência indireta do transtorno mental (diminuição da autoestima devido ao estigma enfrentado ou a fracassos sucessivos no âmbito profissional, motivação diminuída para procurar e manter um trabalho); c) barreiras não relacionadas à doença (escolarização deficitária, atividades ilegais). No âmbito das barreiras externas estão: oportunidades restritas de trabalho, resultantes, muitas vezes, da estigmatização por parte dos empregadores; dificuldades com transporte; suporte social prejudicado; condições precárias de moradia e subsistência, entre outras.

Quanto às formas de atuação que integram os serviços de reabilitação vocacional, destacam-se o treinamento de habilidades relacionadas à recolocação no mercado de trabalho (elaboração de currículos, preparação para entrevistas de empre- gos, busca de vagas disponíveis), aperfeiçoamento educacional, desenvolvimento pessoal e serviços de suporte relacionados ao atendimento das necessidades primárias do paciente, como creche para os filhos, transporte, moradia e alimentação (Karuntzos, 2002).

Em linhas gerais, o protocolo de reabilitação vocacional utilizado nas agências vocacionais inclui: a) avaliação das necessidades vocacionais do paciente; b) planejamento do atendimento vocacional; c) acesso às informações relacionadas ao atendimento para dependência química; d) aconselhamento vocacional individual; e) workshops em treinamento/ motivação para o trabalho; f) atendimento das necessidades primárias do paciente, identificadas como barreiras à sua reabilitação vocacional (Karuntzos, 2002; Platt, 1995).

O tempo adequado ao recebimento dessas intervenções varia conforme a história vocacional do paciente, sua motivação em relação à questão ocupacional e conforme a própria diretriz do serviço de reabilitação vocacional. Existem distintas concepções subjacentes aos programas de reabilitação vocacional. A mais antiga delas surgiu em meados dos anos 1960, nos Estados Unidos, e valoriza o chamado status ocupacional do paciente - uma avaliação sobre a prontidão da pessoa para retornar ao mercado de trabalho. Com base nesta concepção, algumas pessoas são consideradas prontas para ingressar em um programa de reabilitação vocacional, já que possuiriam habilidades, histórico profissional e motivação para imediatamente conseguir trabalho, enquanto outras precisariam de mais atividades pré-vocacionais antes de focalizar uma vaga no mercado de trabalho (Karuntzos, 2002). Esta abordagem prioriza, portanto, as etapas iniciais do processo de reabilitação vocacional (avaliação e intervenções pré-vocacionais), voltadas à motivação e preparação do paciente para o retorno ao mercado de trabalho. As intervenções pré-vocacionais enfatizam trabalhos temporários e/ou voluntários, que ocorrem em ambientes protegidos, constituindo treinamentos vinculados ao serviço vocacional.

A outra abordagem subjacente aos serviços vocacionais intitula-se supported employment e consolidou-se nos Estados Unidos a partir de 1980. 
Esta abordagem enfatiza a importância de um retorno imediato ao mercado de trabalho, priorizando as situações reais, vivenciadas nos cotidianos de trabalho, em detrimento de situações enfrentadas em ambientes protegidos ou mesmo em contextos de trabalho temporário, que envolvem extensos períodos de atividades pré-vocacionais e empregos transitórios e protegidos (Becker e col., 2005; Bond e col., 2001). O foco desta abordagem é a obtenção de emprego no mercado de trabalho formal, em ambientes integrados à comunidade, considerando as potencialidades, recursos, prioridades, habilidades e interesses pessoais do paciente (Bond e col., 2001). Este enfoque prevê um suporte contínuo ao paciente, para além da busca inicial pelo trabalho. Em alguns desses serviços, a ajuda é disponibilizada em tempo real por meio de acesso on-line ao conselheiro vocacional, de maneira que o paciente possa esclarecer dúvidas e buscar orientações sobre como lidar com alguma situação difícil, no momento exato em que ela está ocorrendo. O supported employment é atualmente a abordagem mais utilizada nos serviços vocacionais da América do Norte e da Europa.

Embora relevante ao processo de reabilitação do dependente químico, o que se observa, contudo, é uma oferta escassa de serviços ocupacionais a essa população, considerando-se a demanda existente, mesmo nos países em que essa intervenção está estruturada há décadas (Becker e col., 2005; French e col., 1992). Embora os motivos que justifiquem esse fenômeno não estejam claros, respostas potenciais incluem: conflitos com a filosofia do tratamento, falta de incentivos financeiros aos programas e treinamento e supervisão à equipe inadequados (Platt, 1995; Brewington e col., 1987).

No contexto assistencial brasileiro, inexiste uma intervenção estruturada, segundo a perspectiva esboçada acima, na organização do auxílio ao processo de reabilitação profissional de pessoas com problemas relacionados ao abuso de substâncias. As diretrizes do governo brasileiro para a inclusão social de dependentes químicos e portadores de transtornos mentais pelo trabalho pautam-se nos princípios do cooperativismo, conforme apresentaremos a seguir.

\section{Saúde Mental e Economia Solidária: inclusão social pelo trabalho}

No Brasil, historicamente, a questão do consumo problemático de álcool e drogas foi abordada sob a ótica predominantemente psiquiátrica, focalizando como meta central a abstinência e desconsiderando as implicações sociais, psicológicas, econômicas e políticas desse fenômeno, tal como reconhecido pelo Ministério da Saúde no documento intitulado "A Política do Ministério da Saúde para a Atenção Integral a Usuários de Álcool e outras Drogas" (Brasil, 2004). Uma política nacional de atenção aos usuários de substâncias psicoativas está ainda em consolidação no Brasil e vincula-se estreitamente à política de saúde mental vigente.

A utilização de estratégias de redução de danos, bem como a ênfase em parcerias interministeriais e com setores da sociedade civil organizada, são princípios enfatizados pelo governo brasileiro como fundamentais ao enfrentamento da problemática relacionada ao consumo de substâncias psicoativas, considerando-se a heterogeneidade característica da dependência química e o seu caráter de saúde pública. Dada a complexidade envolvida no fenômeno da dependência de substâncias psicoativas, veicula-se como diretriz oficial uma abordagem intersetorial, envolvendo ministérios do governo, organizações da sociedade civil de interesse público e outras entidades não governamentais, direta ou indiretamente relacionadas ao tema.

No campo específico da reabilitação profissional de dependentes químicos, destaca-se a parceria entre o Ministério da Saúde (MS) e o Ministério do Trabalho e Emprego (MTE), por meio do programa governamental intitulado "Saúde Mental e Economia Solidária: Inclusão Social pelo Trabalho". Filiada aos princípios da reforma psiquiátrica, esta proposta almeja a inclusão social de usuários de serviços de saúde mental e de álcool e drogas por meio da construção de empreendimentos solidários (cooperativas de trabalho autossustentáveis), organizados segundo os princípios da autogestão e administração participativa e democrática, que visam ao trabalho emancipado (Brasil, 20o6). Con- 
forme descrito no Documento Final da I Conferência Nacional de Economia Solidária (Brasil, 2006b), a economia solidária inspira-se em valores culturais que colocam o ser humano em sua integralidade ética, como sujeito e finalidade da atividade econômica, ambientalmente sustentável e socialmente justa, em detrimento da acumulação privada do capital. Trata-se, portanto, de uma alternativa diferenciada de trabalho, cujo incentivo volta-se às iniciativas locais e alternativas ao mercado formal de trabalho. As iniciativas em economia solidária agregam, em geral, populações marcadas pela exclusão, qualquer que seja o fator propiciador desta condição - aspectos socioeconômicos, saúde mental prejudicada, consumo de substâncias psicoativas. Amparado legalmente por uma legislação específica, o cooperativismo está no Brasil a cargo da Secretaria Nacional de Economia Solidária (SENAES), vinculada ao Ministério do Trabalho e Emprego.

Ainda que algumas iniciativas em economia solidária datem de mais de dez anos, o que se observa em geral é a fragilidade das experiências em curso, que parece decorrer de uma sustentação legal e de incentivos financeiros ainda pouco consistentes para promover amparo seguro; essas inciativas carecem, assim, de maior acompanhamento e consolidação. Isto se torna ainda mais premente quanto se considera a população dependente química, já que as iniciativas usualmente em curso congregam, em sua maioria, egressos de serviços de saúde mental. Existem atualmente 640 iniciativas de geração de renda registradas no Cadastro Nacional de Iniciativas de Inclusão Social pelo Trabalho, concentrando-se a maioria delas na região sudeste do País (Brasil, 2011).

\section{À Guisa de Conclusão: dependência química, trabalho e saúde}

Um olhar atento às ações pautadas na reabilitação vocacional e praticadas em âmbito internacional, por um lado, e às diretrizes propostas pelo governo brasileiro fundada nos princípios do cooperativismo e da economia solidária, por outro, permitem apreender distintas concepções de saúde subjacentes às práticas realizadas.

Ao oferecer a populações excluídas por alguma condição clínica crônica auxílio para a inclusão no mercado formal de trabalho, as ações vinculadas à reabilitação vocacional parecem privilegiar o viés da doença e da incapacidade para buscar a inclusão desse grupo em particular. A própria terminologia utilizada pela literatura da área, ao enfatizar a condição de paciente, agrega o valor da doença à população-alvo dos programas vocacionais.

Já as ações propostas em âmbito nacional, ao focalizarem de maneira abrangente populações marcadas pela exclusão, qualquer que seja o fator propiciador da condição desse processo - aspectos socioeconômicos, saúde mental prejudicada, uso de álcool e droga - ultrapassa o aspecto clínico, historicamente contido na compreensão do sofrimento psíquico. Neste sentido, a ênfase do processo de inclusão desloca-se da incapacidade provocada pela doença, para as potencialidades contidas em uma concepção ampliada de saúde e de sujeito. Enquanto a população-alvo das intervenções da reabilitação vocacional é constituída por pacientes de diversos serviços de saúde mental, a população-alvo das iniciativas de geração de renda envolve sujeitos portadores de valores e potencialidades, para além da condição clínica ou social que possam portar. 0 enfoque aqui, sintetizado pelos princípios da reabilitação psicossocial, recai sobre as capacidades do sujeito em questão, a despeito das limitações que possua em decorrência de uma doença ou de uma condição socioeconômica desfavorável.

O próprio caráter de doença contido na terminologia relacionada à dependência química merece atenção. Embora não seja possível aprofundar esta discussão nos limites deste artigo, vale lembrar que o conceito de dependência química circunscreve-se, historicamente, ao campo da medicina, enfatizando o polo relacionado ao indivíduo, cujo consumo de álcool e/ou drogas é considerado disfuncional. Neste sentido, a dependência química, como categoria nosográfica, acaba por vezes por se tornar hegemônica e produtora de mais estigma e discriminação, contribuindo para consolidar o desenvolvimento de identidades e subjetividades pautadas pelo adoecimento. Para além do diagnóstico médico, destaca-se a complexidade envolvida no fenômeno do abuso e dependência de álcool e drogas, cuja dinâmica necessariamente envolve a interação entre o indivíduo, com suas características biológicas e de personalida- 
de, a substância, com seu potencial de toxicidade e o meio ambiente no qual se realiza o encontro entre o indivíduo e a substância psicoativa (Silveira, 1995; Nowlis, 1982). Vale observar, portanto, que o conceito de dependência química, como diagnóstico médico, refere-se a um fenômeno distinto do consumo de substâncias psicoativas, tal como observado desde os primórdios da humanidade em todas as culturas, seja para fins religiosos, medicinais, como forma de contestação social ou simplesmente como fonte de prazer e diversão (Vetulani, 2001).

Além da ênfase no viés da doença, outro aspecto que diferencia as duas abordagens observadas neste artigo diz respeito ao universo do trabalho. As diretrizes do governo brasileiro para a reabilitação profissional de dependentes químicos, ao se pautarem nos princípios do cooperativismo, distinguem-se da prática corrente em âmbito internacional, direcionada prioritariamente para a recolocação no mercado formal de trabalho. Embora distintas em relação à filosofia subjacente e aos recursos utilizados, as duas abordagens convergem em relação ao objetivo de auxiliar a pessoa em recuperação a retomar uma atividade ocupacional significativa, enfatizando o potencial de saúde relacionado ao trabalho.

Contudo, parece-nos importante considerar as características que cercam o trabalho na atualidade. Nas últimas décadas, o contexto socioeconômico que marca o trabalho em grande parte dos países tem produzido condições um tanto adversas aos indivíduos, repercutindo diretamente sobre a subjetividade dos trabalhadores. Assim, embora permaneça como atividade central na vida humana, o trabalho, em diversos contextos, tornou-se uma atividade aviltada e estranha para aquele que o realiza (Antunes, 200o). Este fato fica mais potencializado diante do crescente desemprego estrutural e da precarização das condições de trabalho - características evidentes em âmbito mundial, e não somente nas economias de países em desenvolvimento, como o Brasil (Antunes, 200o). À diminuição radical do trabalho assalariado estável soma-se, como fenômeno crescente, a terceirização da mão de obra, cada vez mais responsável pela produção e cada vez mais precária no tocante aos direitos (Antunes, 1999). À terceirização somase também o aumento do trabalho sem registro em carteira e do trabalho como autônomo. Dados prove- nientes do IBGE indicavam, já no final da década de 1990, uma redução significativa de trabalhadores na indústria, em contrapartida ao aumento substancial do número de pessoas trabalhando nos setores de prestação de serviços e de comércio. Simultaneamente, já se delineava a redução significativa de trabalhadores com carteira assinada e o aumento do número de pessoas trabalhando sem registro em carteira e por conta própria (Instituto Brasileiro de Geografia e Estatística, 2005).

Tais fatos contribuem sobremaneira para aumentar a fragilidade dos vínculos sociais, uma característica marcante na contemporaneidade. Segundo destaca Sennett (1999), o rompimento, na atualidade, da noção de longo prazo, somado ao desejo de retorno rápido e à estruturação das organizações em redes - mais planas e flexíveis - ao invés das antigas hierarquias piramidais - mais estáveis e difíceis de serem decompostas ou redefinidas - repercutiu diretamente sobre a possibilidade de se estabelecer confiança, atributo relacionado às relações duradouras. Segundo o autor, o esquema de curto prazo característico das instituições modernas limita a possibilidade de amadurecimento da confiança, já que os vínculos fortes dependem da associação a longo prazo e da disposição em estabelecer compromissos com os outros (Sennett, 1999). Mas como é possível desenvolver confiança e uma narrativa de identidade e história de vida em uma sociedade composta por episódios e fragmentos?

Nesse contexto, evidencia-se o quanto o trabalho não é neutro em relação à saúde, podendo operar tanto estados de saúde e prazer, quanto estados de infelicidade e doença (Dejours, 1992), de modo que o desempenho de uma atividade profissional não garante, por si, a emancipação do sujeito que o realiza. De maneira geral, essa discussão sobre a relação trabalho-saúde parece não estar contemplada na literatura sobre reabilitação vocacional.

Com base nesse panorama, é possível dimensionar a complexidade do processo de reabilitação para dependentes químicos, pois são necessárias intervenções que se estendam no tempo, auxiliando a pessoa em recuperação a construir um projeto de vida, vinculado à constituição de uma identidade ocupacional, e não apenas ao desempenho de uma tarefa. Se o objetivo é favorecer a autonomia da pes- 
soa em recuperação, torna-se imprescindível que a intervenção em reabilitação profissional considere o trabalho, não como terapêutica laboral, mas em sua dimensão emancipatória, como possibilidade de construção do mundo e da subjetividade humana (Ribeiro, 2004). Focalizar, portanto, o retorno a uma atividade formal de trabalho ou a inclusão do sujeito em uma cooperativa de trabalho não garantem, necessariamente, o objetivo de reabilitação dos dependentes químicos. É preciso que a reabilitação para o trabalho integre o processo ampliado de reabilitação psicossocial da pessoa prejudicada pela dependência química, constituindo, antes, uma estratégia de reabilitação pelo trabalho. Caso contrário, corre-se o risco de que o trabalho contribua para produzir ou agravar estados de adoecimento, em oposição ao desejado propósito de promoção de saúde.

\section{Referências}

ANTUNES, R. O mundo precarizado do trabalho e seus significados. Cadernos de Psicologia Social do Trabalho, São Paulo, v. 2, n. 1, p. 55-72, 1999.

. Adeus ao trabalho? Ensaio sobre as

metamorfoses e a centralidade do mundo do trabalho. São Paulo: Cortez, 2000.

BECKER, D. R.; DRAKE, R. E.; NAUGHTON, W. J. Supported employment for people with cooccuring disorders. Psychiatric Rehabilitation Journal, Boston, v. 28, n. 4, p. 332-338, 2005.

BOND, G. R. et al. Implementing supported employment as an evidence-based practice. Psychiatric Services, Washington, DC, v. 52, n. 3, p. 313-322, 2001.

INSTITUTO BRASILEIRO DE GEOGRAFIA E ESTATÍSTICA. Educação e trabalho. Análise dos resultados [citado em 03 jun. 2005]. Disponível em: <http://www.ibge.gov.br/estatistica>. Acesso em: 01 dez. 2009.

BRASIL. Ministério da Saúde. Secretaria de Atenção à Saúde. SVS/CN-DST/AIDS. A política do ministério da saúde para atenção integral a usuários de álcool e outras drogas. Brasília, DF, 2004 .
BRASIL. Ministério da Saúde. Secretaria de Atenção à Saúde - Departamento de Ações Programáticas Estratégicas. Saúde mental e economia solidária - inclusão social pelo trabalho. Brasília, DF, 20o6a.

BRASIL. Ministério do Trabalho e Emprego. Economia solidária como estratégia e política de desenvolvimento. Brasília, DF, 2006b.

BRASIL. Ministério da Saúde. Saúde mental em dados. Brasília, DF, 2011. Disponível em: <www. saude.gov.br/bvs/saudemental $>$. Acessado em: 15 jan. 2012.

BRAVERMAN, H. Trabalho e capital monopolista - a degradação do trabalho no século XX. Rio de Janeiro: Zahar, 1981.

BREWINGTON V. et al. Obstacles to the utilization of vocational services: an analysis of the literature. International Journal of the Addictions, New York, NY, v. 22, n. 11, p. 1091-1118, 1987.

COMERFORD, A. W. Work dysfunction and addiction: common roots. Journal of Substance Abuse Treatment, New York, NY, v. 16, n. 3, p. 247253, 1999.

CENTER FOR SUBSTANCE ABUSE TREATMENT. Integrating substance abuse treatment and vocational services. Treatment Improvement Protocol Series, Rockville, MD, n. 38, 2000.

DEJOURS, C. A loucura do trabalho: estudo de psicopatologia do trabalho. São Paulo: Cortez/ Oboré, 1992.

DEREN, M. P.; RANDELL, J. The vocational rehabilitation of substance abusers. Journal of Applied Rehabilitation Counseling, Washington, DC: v. 21, n. 1, p. 3-6, 1990.

EDWARDS, G.; MARSHALL, E. J.; COOK, C. C. H. O tratamento do alcoolismo: um guia para profissionais da saúde. Porto Alegre: Artmed, 1999.

\section{EFFECTIVE INTERVENTIONS UNIT. Moving} on: education, training, and employment for recovering drug users. Research Review. Scotland, 2001. 
FERRETTI, C. J. Uma nova proposta de orientação profissional. São Paulo: Cortez, 1997.

FRENCH, M. T. et al. Training and employment programs in methadone treatment: clients needs and desires. Journal of Substance Abuse Treatment, New York, v. 9, n. 4, p. 293-303, 1992.

KARUNTZOS, G. T. Vocational status as a moderator of substance abuser's employability. 2002. Tese (Doctor of Philosophy) - Faculty of North Carolina State University, North Carolina State.

LAUDET, A. B.; WHITE, W. What are your priorities right now? Identifying service needs across recovery stages to inform service development. Journal of Substance Abuse Treatment, New York, v. 38, n. 1, p. 51-59, 2010.

MAGURA, S. et al. The effectiveness of vocational services for substance users in treatment.

Substance Use \& Misuse, Monticello, NY, v. 39, n. 13-14, p. 2165-2213, 2004.

MOWBRAY, C. T. et al. Enhancing vocational outcomes for persons with psychiatric disabilities: a new paradigm. In: HENGGLER, S.W.; SANTOS, A.B. Innovative approaches for difficult-to-treat populations. Washington: American Psychiatric Press, 1997. p. 311-348.

NATIONAL INSTITUTE ON DRUG ABUSE. Principles of drug addiction treatment: a research-based guide. Rockville, MD: NIDA. NHI Publication, n. 99-4180, 1999.
NOWLIS H. A verdade sobre as drogas. Rio de Janeiro, RJ: IBECC/Unesco, 1982.

PLATT, J. J. Vocational rehabilitation of drug abusers. Psychological Bulletin, Washington, DC, v. 117, n. 3, p. 416-433, 1995.

RIBEIRO, M.A. Orientação Profissional para "pessoas psicóticas": um espaço para o desenvolvimento de estratégias identitárias de transição através da construção de projetos. 2004. Tese (Doutorado em Psicologia Social) - Instituto de Psicologia da Universidade de São Paulo, São Paulo.

ROOM, J. A. Work and identity in substance abuse recovery. Journal of Substance Abuse Treatment, New York, v. 15, n. 1, p. 65-74, 1998.

SENNETT, R. A corrosão do caráter: as consequências pessoais do trabalho no novo capitalismo. Rio de Janeiro: Record, 1999.

SILVEIRA, D. X. Drogas: uma compreensão psicodinâmica das farmacodependências. São Paulo: Casa do Psicólogo, 1995.

VETULANI J. Drug Addiction Part I. Psychoactive substances in the past and present. Polish Journal of Pharmacology, Kraków, Poland, v. 53, n. 3, p. 201-214, 2001. 